\title{
Can headless screw used in fixation of the scaphoid proximal pole fracture be broken after wrist trauma? An unreported complication of scaphoid surgery
}

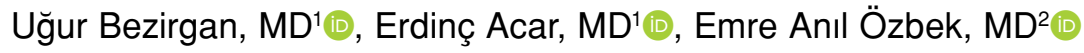 \\ 'Department of Orthopedics and Traumatology, Division of Hand Surgery, Ankara City Hospital, Ankara, Turkey \\ ${ }^{2}$ Department of Orthopedics and Traumatology, Ankara University School of Medicine, Ankara, Turkey
}

The scaphoid is the most frequently fractured carpal bone. Complications of delayed union, nonunion, and osteonecrosis of the proximal pole are well documented in the orthopedic literature. ${ }^{[1]}$ Overall complication rates of surgical fixation of scaphoid fractures vary widely from 0 to $29 \%$, with most studies reporting very low complication rates. One recent report of Bushnell et al. $^{[2]}$ found a major complication (nonunion, misplaced screws, and proximal pole fracture) rate of $21 \%$ and a minor complication (intraoperative guidewire breakages) rate of $8 \% .{ }^{[2]}$

This case report is the first case of headless screw, which was placed for a proximal pole scaphoid fracture, broken three months after primary surgery. The cause of screw breakage was probably fall on an

Received: March 21, 202

Accepted: April 052021

Published online: November 19, 2021

Correspondence: Emre Anıl Özbek, MD. Ankara Üniversitesi Tıp Fakültesi, Ortopedi ve Travmatoloji Anabilim Dalı,

06590 Çankaya, Ankara, Türkiye.

E-mail:anl_ozbek@hotmail.com

Doi: $10.52312 / j d r s .2021 .101$

Citation: Bezirgan U, Acar E, Özbek EA. Can headless screw used in fixation of the scaphoid proximal pole fracture be broken after wrist trauma? An unreported complication of scaphoid surgery. Jt Dis Relat Surg 2021;32(3):779-785.

(2021 All right reserved by the Turkish Joint Diseases Foundation

This is an open access article under the terms of the Creative Commons Attribution-NonCommercial License, which permits use, distribution and reproduction in any medium, provided the original work is properly cited and is not used for commercial purposes (http://creativecommons.org/licenses/by-nc/4.0/).

\section{ABSTRACT}

The post-traumatic impairment of blood supply of the scaphoid bones' proximal pole is the subject of controversy in the surgical techniques which would heal this fracture. In a surgery performed with a dorsal approach, the main goal is to make a strong fixation with a small incision and a good implant without disturbing the blood supply of the bone. To date, complications related to surgical treatment of scaphoid proximal pole fractures have been reported in detail. However, there is no other study which presents headless screw failure which placed for the scaphoid proximal pole fracture surgery. Although implant technologies have been developed nowadays, our case report highlights that the endurance of these implants has not been carried out in such proximal pole fractures of the scaphoid, yet. Herein, we present the explanation of a broken headless screw which was placed into the proximal pole fracture of scaphoid, avoiding to affect bone stock of the scaphoid surgical technique. We believe that this surgical technique would be helpful to manage similar difficult situations for orthopedic surgeons.

Keywords: Implant removal; proximal pole of scaphoid fracture, screw failure.

outstretched hand and also nonunion of the scaphoid bone. The patient was treated with a new surgical technique of screw removal, simple curettage, and local bone graft obtained from the dorsal aspect of the adjacent distal radius and finally fixation with a larger antegrade headless screw.

\section{CASE REPORT}

A 22-year-old otherwise healthy, right-hand dominant machine male technician was referred to our center one week after injuring his left wrist. His medical history revealed that the trauma was in the form of falling on the outstretched hand with the forearm in pronation. He had an operation in an external center 
almost three months ago and the pain and limitation of movement started due to falling on the wrist one week ago. Physical examination was significant for tenderness on the dorsoradial aspect of the scaphoid of the left wrist. His active range of motion was $30^{\circ}$ extension, $40^{\circ}$ flexion, $20^{\circ}$ radial deviation, and $30^{\circ}$ ulnar deviation. Plain X-ray and computed tomography (CT) images at the time of evaluation showed an unhealed proximal pole fracture and a broken headless screw (Figure 1). Also, it was suspected that there might be an ulnar translation of the carpus following the last trauma. Although there was no physical examination finding suggestive of wrist instability, previous radiographs of the patient were requested. Relevant information was obtained based on the previous radiological images, which were performed in another center prior to primary surgery (Figure 2). Accordingly, the carpus gradually moved to the ulna along the fracture line with the first trauma and in follow-up images. The patient was followed with a short scaphoid cast for the first eight weeks and, when the fracture was displaced, surgery was decided. The surgery was performed dorsally with the headless screw, the plaster application was continued for an additional one month. The headless screw inside the scaphoid bone, which did not heal, was broken due to a newly added trauma three months after the operation. Therefore, the patient was referred to our hospital.

The patient was placed in a removable long opponens splint and revision surgery was planned, after the informed consent form was obtained from the patient. Surgery was performed by two certified orthopedic hand surgeons and follow-up was conducted by these two surgeons. Distal radius vascularized bone grafts were not preferred, as the old incision was longitudinal from dorsal and the pedicle might be compromised at initial surgery.

The initial incision was used extending $2 \mathrm{~cm}$ proximally. The extensor pollicis longus tendon was taken out of the extensor compartment and rolled over to the radial side. The joint capsule was opened a L-shaped flap. The wrist was flexed and the proximal end of the headless screw was easily removed with a screwdriver. The non-healed bone region was distracted with manual traction and the distal end of the screw embedded into the bone was presented. A 1.2-mm Kirschner wire (K-wire) was driven with a drill to tighten into a $2.5-\mathrm{mm}$ broken part of the headless screw. As the diameter of the K-wire was slightly larger than the inner diameter of the screw, the K-wire stuck into the screw. The drill was, then, turned in the opposite direction and the screw was removed (Figure 3). Thus, the removal of the implant in the distal pole was performed without impairing the circulation of the scaphoid. Pure cancellous autograft bone was harvested from the distal radius through a dorsal window just proximal to Lister's tubercle. The autograft was compressed in a syringe and, then, packed into the cavity within the bone with a small impactor. A more antegrade central fixation was carried out with a 2.8-mm headless screw (Acutrak ${ }^{\circledR}$; Acumed LLC, OR, USA) after the bone grafting was completed (Figure 4).

The Colles cast was applied for two weeks postoperatively and wrist rehabilitated after cast removal. The tenderness of wrist which was originated

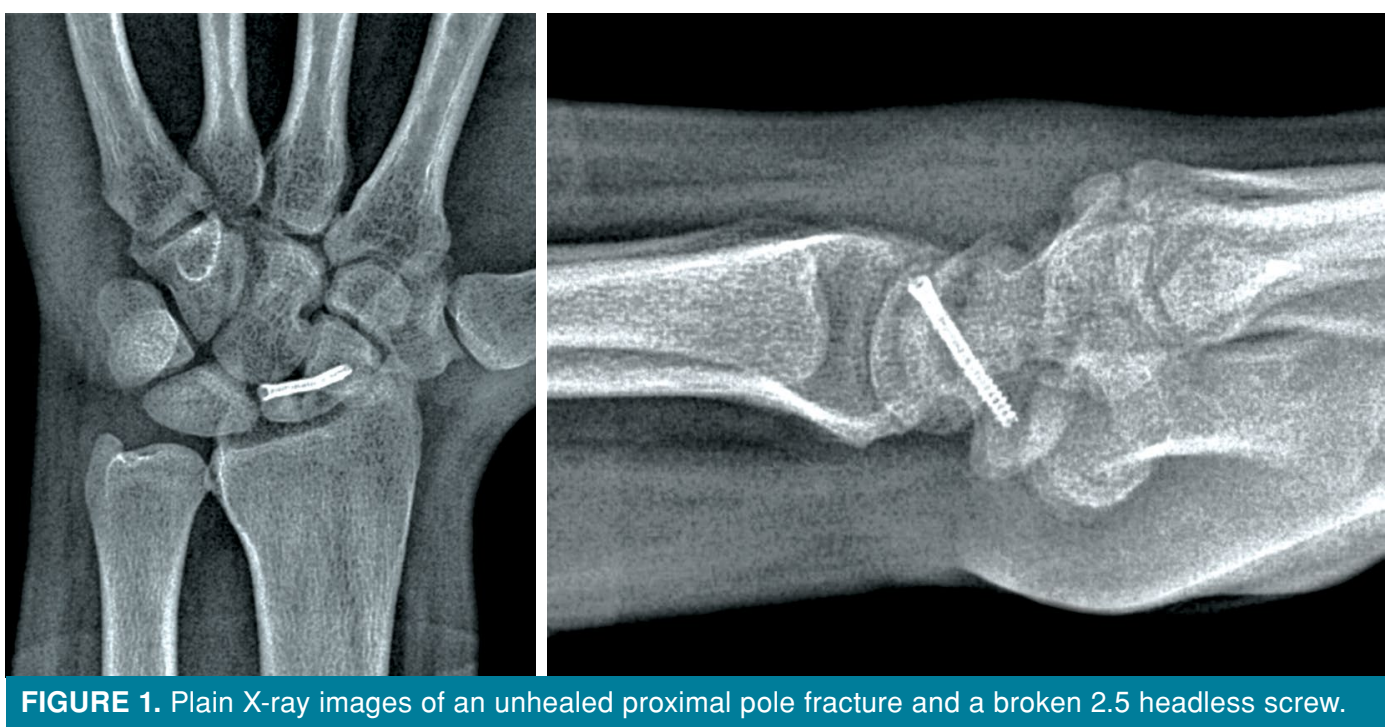



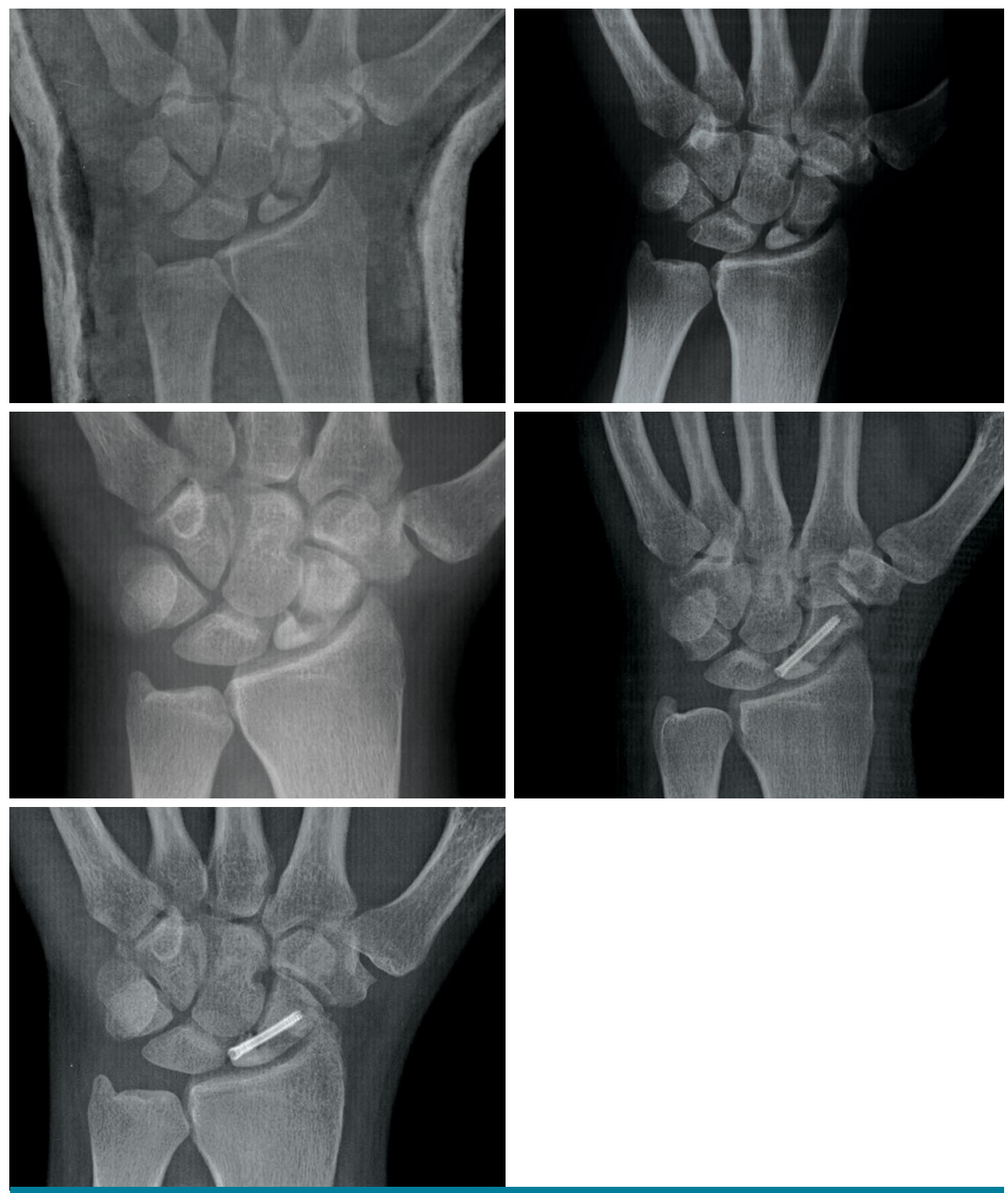

FIGURE 2. Sequential follow-up images at Months 1 to 3.

from non-healed scaphoid bone disappeared after three months and wrist extension was reported as $60^{\circ}$ and flexion as $40^{\circ}$. At three months, there were radiographic signs of healing in the nonunion area without bone resorption (Figure 5). The patient, who was followed for 14 months after surgery, returned to work five months after the last surgery with a good functional condition. The bridging trabecula was found on CT scans in two planes at nine months (Figure 6).

\section{DISCUSSION}

Poor healing which is associated with scaphoid bones' proximal pole fractures are likely to be multifactorial. The proximal pole of the scaphoid is poorly vascularized, with the majority of its perfusion supplied by the dorsal ridge vasculature by retrograde flow. ${ }^{[2]}$ The proximal fragment also tends to be very small, which complicates the fixation of the scaphoid bone. Roughly one-third of acute proximal pole fractures go on to nonunion, when treated non-operatively. ${ }^{[2]}$

There are several options for the treatment of a proximal pole scaphoid nonunion. Due to the difficulty in achieving union, several vascularized bone grafts have been described ${ }^{[3]}$ Earlier reports claimed that proximal pole nonunions were not manageable with cancellous bone grafting; however, 


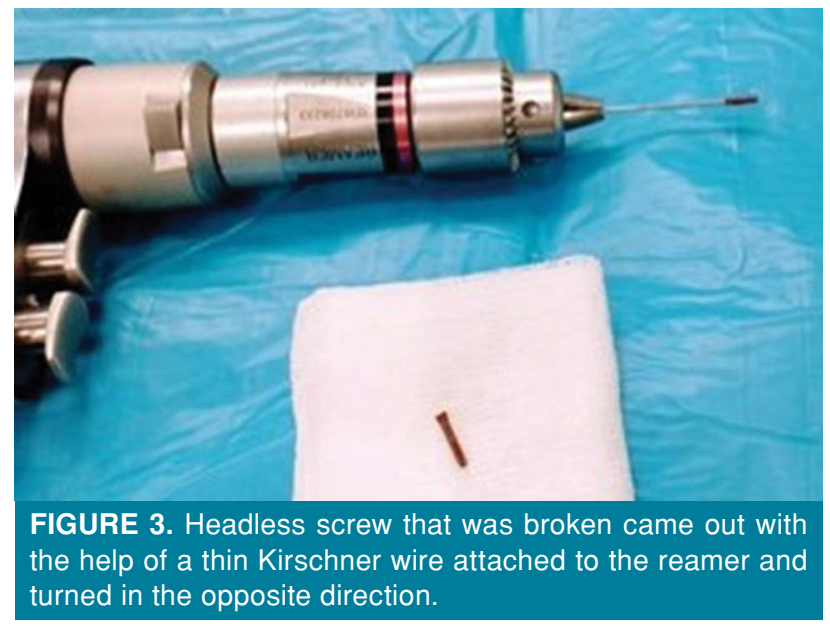

there is a paradigm shift with modern fixation techniques. ${ }^{[4]}$ Luchetti et al. ${ }^{[5]}$ presented 20 patients with established proximal pole scaphoid nonunions treated with curettage and cancellous autograft from the distal radius and screw fixation. Bone union was achieved in 18 of 20 patients $(90 \%)$ as evidenced by CT imaging. Two nonunions that did not heal were treated with repeat curettage and debridement and iliac crest bone grafting without revision of fixation. The authors concluded that applying new implants with the right technique allowed to achieve satisfactory results even in proximal pole fractures of the scaphoid bone. In our case, the reason for not using vascularized dorsal bone grafts was that the initial incision was also made of dorsal which could
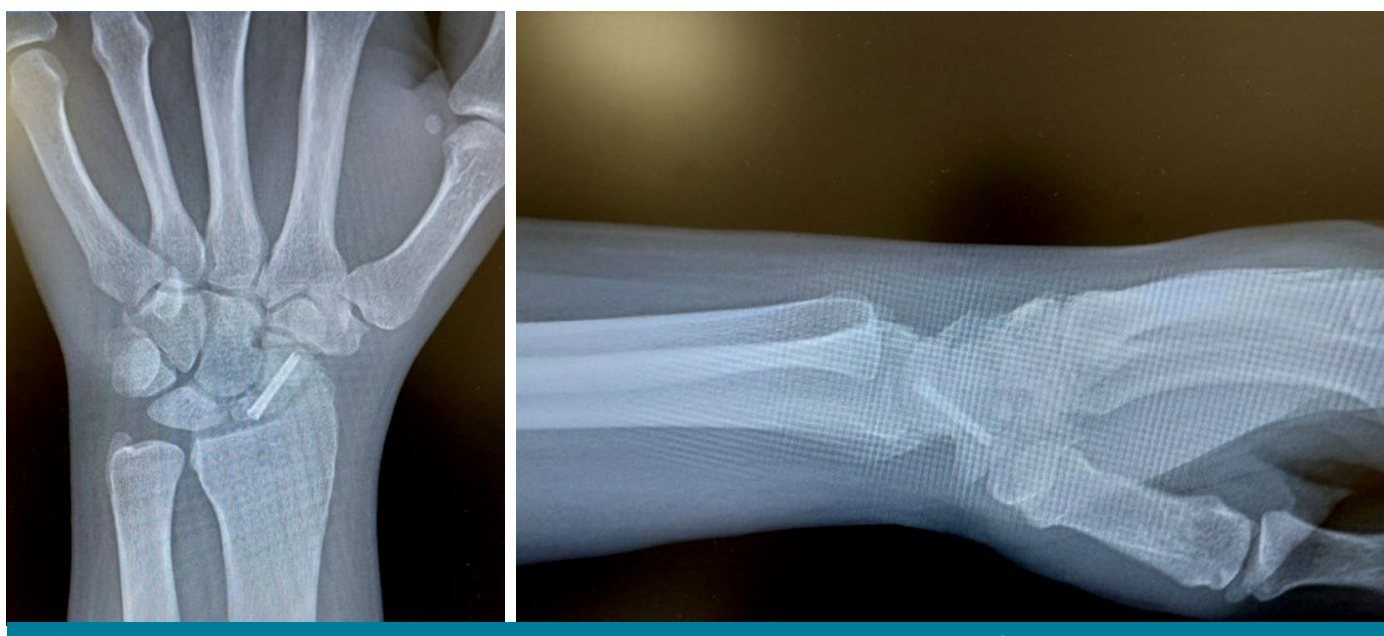

FIGURE 4. Postoperative images of a stable fixation with a $2.8-\mathrm{mm}$ Acutrak ${ }^{\circledR}$ screw $^{-}$
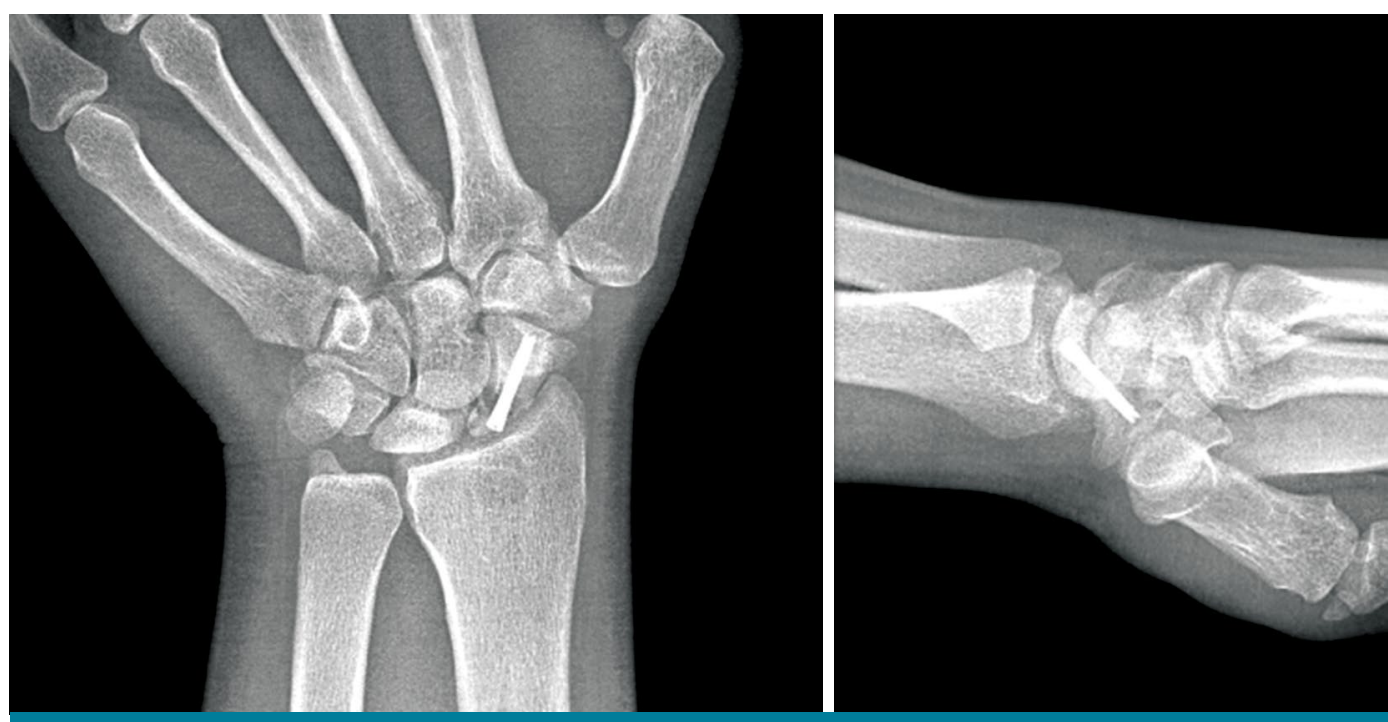

FIGURE 5. Plain radiographs showing new consolidated bone areas in nonunion zone at Month 3. 

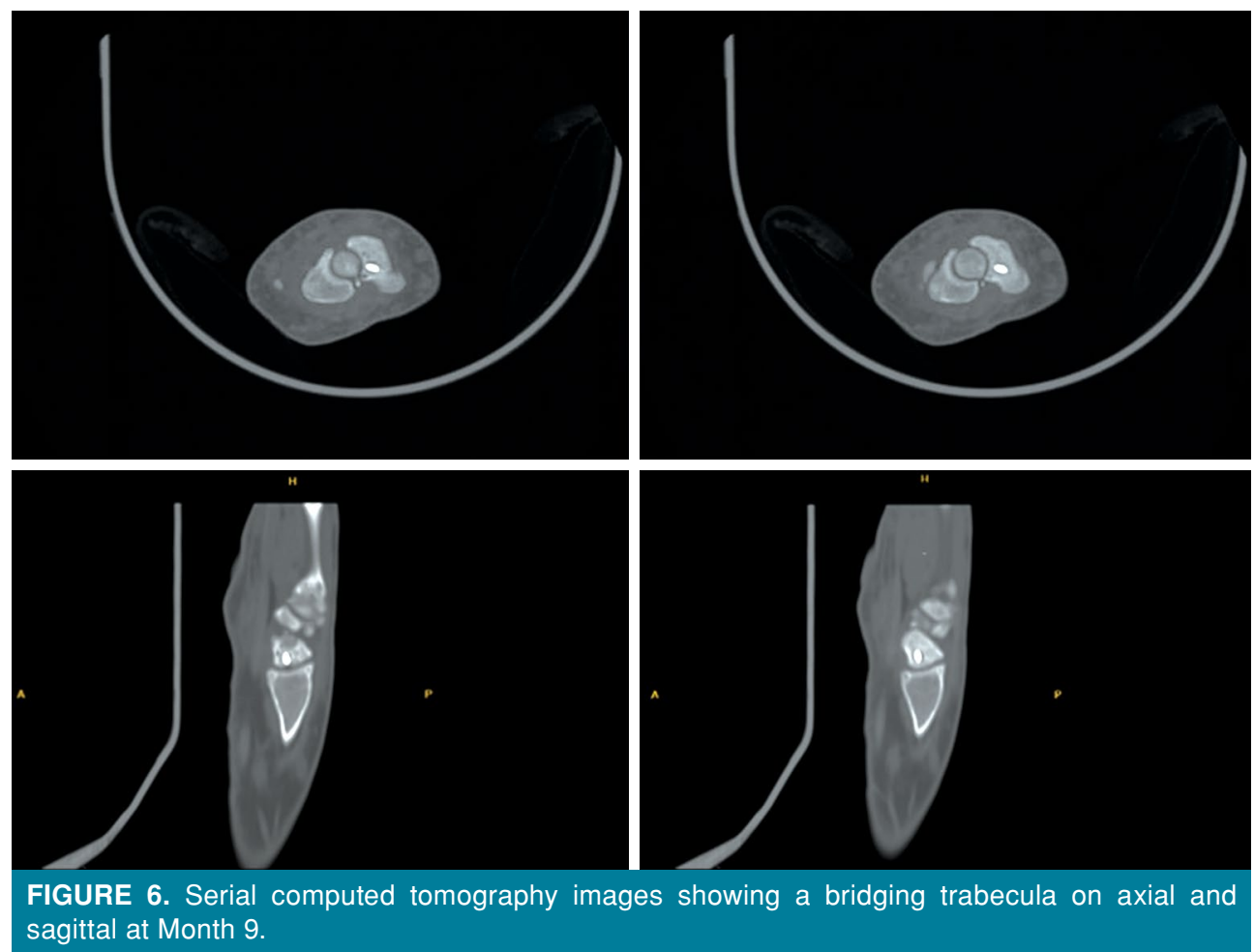

affect the pedicle. We did not use iliac bone graft to prevent an additional morbidity to the patient who was sufficiently traumatized.

In the present case, the initial displacement of the carpus to the ulnar side indicated an injury to the palmar/dorsal radiocarpal ligaments, and non-specific attenuation of the radiocarpal capsule was also considered as previously described. ${ }^{[6]}$ The aim was to improve scaphoid nonunion, as there was no instability on physical examination, and enough time had already passed for soft tissue healing with immobilization.

Fixation with screws are shown to provide more successful union rates than fixation with K-wires. ${ }^{[7]}$ However, it is still controversial which screw type is more suitable for proximal pole nonunion. There are two types of headless compression screws which are mostly used in our clinic. The Herbert ${ }^{\mathrm{TM}}$ (Zimmer Medizin Systems Corp., IN, USA) screws with threads running in the same direction is one of them, although the proximal portion has a wider pitch to its thread. Thus, when the proximal threads engage in the bone, they tend to move through the bone faster than the threads at the distal end, leading to the two ends of the bone to compress together. The Acutrak ${ }^{\circledR}$ screws are cannulated and headless screws, which allow it to be implanted below the surface of the bone. These screws use the same concept of variable thread pitch as the Herbert $^{\mathrm{TM}}$ screws; however, unlike the Herbert ${ }^{\mathrm{TM}}$ screws, they are fully threaded, with continuously varying pitch along their length. This variable pitch creates constant compression across a fracture as the screw is advanced, and gives the screw its unique appearance. This feature may improve internal holding power, as well as allow a fracture or osteotomy site to lie anywhere along the length of the screw. ${ }^{[8]}$ Despite the difference in compression between Acutrak ${ }^{\circledR}$ and Herbert ${ }^{\mathrm{TM}}$ screws, functional outcomes and time to bone healing are comparable in scaphoid proximal pole nonunion..$^{[9]}$

Propagation of a fracture at the site of screw insertion is one of the major complications reported after scaphoid's proximal pole fracture surgery. ${ }^{[5]}$ The variable pitch cannulated headless Acutrak $^{\circledR}$ screw placed without the use of the second drill bit (profile drill), particularly in the proximal pole fractures, is particularly vulnerable. In cases of scaphoid nonunion with reduced vascularity, proximal bone may be weakened and, thus, unable to withstand the pressure load on the scaphoid. Simply placing the screw after drilling with the standard long drill bit can generate an excessive hoop stress, leading to iatrogenic fracture line propagation from the screw entry point during the insertional torque. Therefore, 
some authors recommend the Herbert ${ }^{\mathrm{TM}}$ screw instead of Acutrak $^{\circledR}$ while fixing the proximal pole fracture of the scaphoid. ${ }^{[7,10]}$ In our case, it is worth mentioning that, if we had a Herbert ${ }^{\mathrm{TM}}$ screw, we would definitely use it. In such a case, what is more important than the choice of the screw type is a rigid fixation to be made with a bone graft without creating a new fracture line.

The fact that the $2.5 \mathrm{~mm}$ headless screw was broken compelled us to use a larger compression screw. We preferred Acutrak ${ }^{\circledast}$ of $2.8 \mathrm{~mm}$ and turned the screw driver very slowly and controlled while burying the head under the cartilage. To the best of our knowledge, for the first time in the literature, the patient was treated with a new surgical technique of screw removal, simple curettage, and local bone graft obtained from the dorsal aspect of the adjacent distal radius and finally fixation with a larger antegrade headless screw. We consider that the quality of the broken screw and the material produced or the inadequate insertion of the threaded bone screw may cause this complication.

In the initial surgery, it was easily detected that the screw was placed slightly eccentrically on the capitate side of the proximal scaphoid. The screw that was not placed centrally in the proximal fragment had to bear almost all of the loads on the scaphoid bone. In this case, a misplaced screw may have been overloaded and fatigue may have developed over time. Central placement of the screw in the proximal fragment of the scaphoid had superior results, compared to those after eccentric positioning of the screw. ${ }^{[11]}$ Hence, every effort and technique should be applied with care to place a central screw in scaphoid fractures.

The only available data regarding the implant written in the surgery report is that this screw is a 2.5-mm headless compression screw without a trademark or brand name. Therefore, we are curious about whether this screw was broken due to a failure in the production phase. Any study was found about biomechanical study of the screw, which was placed in the initial surgery. Due to these issues, the following question could not be answered in our case: Was the screw breakage due to metallic fatigue or did the sudden forces acting on scaphoid with the newly added trauma exceed the endurance strength of the implant?

Insertion of a small or large screw from the dorsal scaphoid should be also discussed. Among the headless screws we use, the small screws are longer than the wider ones. Although screws with a smaller diameter are longer, a smaller diameter can have an adverse effect on fixation strength. Biomechanical studies have shown that wider screws provide better resistance to lateral displacement forces, as their resistance is proportional to the radius of the screw to the fourth power. ${ }^{[12]}$ This is exactly why we used a screw that was wide and long enough for the revision of this case.

It is well known that the scaphoid bone is the narrowest at the proximal pole. Although large screws are more durable, there is a handicap in demonstrating $\mathrm{CT}$ recovery in proximal fractures of the scaphoid. If a wide screw is placed in a narrow area, it may not be always possible to show the bridging trabeculae, as it is expected. The use of larger implants may result in less bridging trabeculae.

In conclusion, implants and application technique used in the scaphoid proximal pole fractures seem to overcome difficulties and fatigue of microsurgical techniques. Consequently, a stable and load-bearing fixation respecting the biology would always heal the bone with minimal damage. Also, we believe that this surgical technique would be helpful to manage similar difficult situations for orthopedic surgeons.

\section{Declaration of conflicting interests}

The authors declared no conflicts of interest with respect to the authorship and/or publication of this article.

\section{Funding}

The authors received no financial support for the research and/or authorship of this article.

\section{REFERENCES}

1. Acar B, Köse Ö, Turan A, Katı YA, Güler F. Single versus double screw fixation for the treatment of scaphoid waist fractures: Finite element analysis and preliminary clinical results in scaphoid nonunion. Jt Dis Relat Surg 2020;31:73-80.

2. Bushnell BD, McWilliams AD, Messer TM. Complications in dorsal percutaneous cannulated screw fixation of nondisplaced scaphoid waist fractures. J Hand Surg Am 2007;32:827-33.

3. Bürger HK, Windhofer C, Gaggl AJ, Higgins JP. Vascularized medial femoral trochlea osteocartilaginous flap reconstruction of proximal pole scaphoid nonunions. J Hand Surg Am 2013;38:690-700.

4. Kawamura K, Chung KC. Treatment of scaphoid fractures and nonunions. J Hand Surg Am 2008;33:988-97.

5. Luchetti TJ, Rao AJ, Fernandez JJ, Cohen MS, Wysocki RW. Fixation of proximal pole scaphoid nonunion with nonvascularized cancellous autograft. J Hand Surg Eur Vol 2018;43:66-72.

6. Rayhack JM, Linscheid RL, Dobyns JH, Smith JH. Posttraumatic ulnar translation of the carpus. J Hand Surg Am 1987;12:180-9. 
7. Rancy SK, Zelken JA, Lipman JD, Wolfe SW. Scaphoid proximal pole fracture following headless screw fixation. J Wrist Surg 2016;5:71-6.

8. Loving VA, Richardson ML. Scaphoid fracture fixation with an Acutrak(®) screw. Radiol Case Rep 2015;1:58-60.

9. Gereli A, Nalbantoglu U, Sener IU, Kocaoglu B, Turkmen $\mathrm{M}$. Comparison of headless screws used in the treatment of proximal nonunion of scaphoid bone. Int Orthop 2011;35:1031-5.
10. Gaston RG, Chadderdon RC. Management of complications of wrist fractures. Hand Clin 2015;31:193-203.

11. McCallister WV, Knight J, Kaliappan R, Trumble TE. Central placement of the screw in simulated fractures of the scaphoid waist: A biomechanical study. J Bone Joint Surg [Am] 2003;85:72-7.

12. Toby EB, Butler TE, McCormack TJ, Jayaraman G. A comparison of fixation screws for the scaphoid during application of cyclical bending loads. J Bone Joint Surg [Am] 1997;79:1190-7. 